\title{
Robust Channel Estimation Methods for Spectrally Efficient FDM Systems
}

\author{
Hedaia Ghannam and Izzat Darwazeh \\ Department of Electrical and Electronics Engineering \\ University College London, London, United Kingdom \\ E-Mail: hedaia.ghannam.15@ucl.ac.uk, i.darwazeh@ucl.ac.uk
}

\begin{abstract}
This paper proposes and explores a novel channel estimation scheme for non-orthogonal multi-carrier signals and systems; spectrally efficient frequency division multiplexing (SEFDM), in which higher spectral efficiency is achieved by violating the orthogonality of its subcarriers. The proposed scheme is distinguished by its simplicity, low computational complexity, high accuracy and performance independent of the number of subcarriers and compression factor. The presented results demonstrate the efficacy of the proposed scheme by comparing its complexity and performance to other estimation schemes.
\end{abstract}

\section{INTRODUCTION}

Improving the spectral efficiency is a key challenge in the design of wireless communication systems and a major consideration in 5th generation systems and beyond [1], [2]. Many techniques were proposed for the purpose of improving spectral efficiency relative to that of the well known orthogonal frequency division multiplexing (OFDM). SEFDM was originally proposed in 2003 [3] and the idea behind it is to pack more subcarriers than OFDM in a given bandwidth. Despite of the non-orthogonality, techniques generally used in wireless systems, such as channel coding, equalisation and detection have been applied to SEFDM. Indeed, significant spectral efficiency gains were demonstrated in experimental SEFDM test beds for wireless [4], optical/mm-wave [5], VLC system [6] [7], very high speed optical [8] and the downlink for Internet of Things (IoT) networks [9].

The non-orthogonality nature of SEFDM signals further complicates the channel estimation problem. Two time domain channel estimation schemes for SEFDM systems were proposed in [10] and another one in [6]. These schemes show acceptable performance, however, their complexity limits their practical utilisation because they require matrix inversions. To estimate the channel in the frequency domain, the work in [8] divides the transmitted block into orthogonal and nonorthogonal multiplexing regions. The orthogonal region is used specifically for channel estimation based on least squares (LS) [8]. However, this scheme suffers from the disadvantage of interpolation, that increases the estimation complexity and reduces its accuracy, as the frequencies of subcarriers in OFDM region are not the same as SEFDM region [8]. Recently, a joint iterative channel estimation and equalisation scheme in the frequency domain is proposed for singlecarrier non-orthogonal faster-than-Nyquist (FTN) systems, where a trace (trellis) operation is used to minimize the estimation error [11]. Although the estimation accuracy is enhanced gradually in each iteration, the complexity and delay due to the estimator iterative nature limit the scheme practicality.

In this paper, we exploit the compressed subcarriers feature of SEFDM systems to design a novel frequency domain channel estimation scheme.The proposed scheme demonstrates substantial performance and computational complexity gains over other existing schemes.

\section{SEFDM SignALS OVER MULTIPATH CHANNELS}

SEFDM is a multi-carrier modulation technique, where symbols are generated similarly to OFDM by means of modified IFFT structures [4]. Let $\mathbf{z} \in \mathbb{C}^{N \times 1}$ be a complex QAM baseband symbol of duration $T_{s}$, to be modulated by SEFDM in a multicarrier multiplexing manner, then the $k^{\text {th }}$ SEFDM-modulated symbol is presented as [4]

$$
x_{k}(t)=\sum_{n=0}^{N-1} z_{k, n} \exp (2 \pi j n \alpha \Delta f(t-k T)) \text {, }
$$

where $N$ denotes the number of subcarriers, $z_{k, n}$ is the QAM symbol modulated by the $n^{\text {th }}$ subcarrier, $T=N \times T_{s}$ is the SEFDM signal duration and $\Delta f=1 / T$ is the orthogonal frequency spacing between subcarriers. $\alpha \in(0,1]$ is the compression factor, which indicates bandwidth savings of $((1-\alpha) / \alpha) \times 100 \%$ when compared to $\operatorname{OFDM}(\alpha=1)$. 


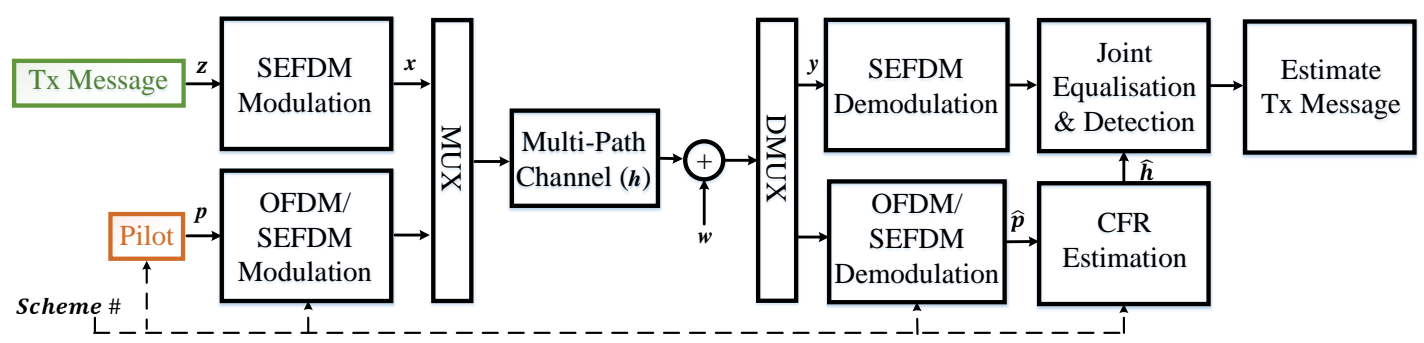

Fig. 1: Transceiver block diagram.

If the signal in (1) were to be transmitted over a frequency-selective multipath channel of $L$ paths, the received signal can be expressed as

$$
y_{k}(t)=\sum_{L} \gamma_{l} x_{k}\left(t-\tau_{l}\right)+w_{k}(t)
$$

where $\gamma_{l}, \tau_{l}$ are the complex amplitude and delay of the $l^{\text {th }}$ path, respectively. $w_{k}$ is the additive white Gaussian noise (AWGN), with zero mean and variance $\sigma_{n}^{2}=N_{0} / 2$, where $N_{0}$ is the noise power spectral density.

At the receiver, the estimated symbol at the $n^{t h}$ subcarrier $\hat{z}_{k, n}$ affected by the channel and contaminated by interference resulting from the non-orthogonal nature of the SEFDM signal can be expressed as

$$
\begin{aligned}
\hat{z}_{k, n} & =y_{k}(t) \exp (-2 \pi j n \alpha \Delta f t) \\
& =\sum_{L} \gamma_{l}\left[\sum_{m=0}^{N-1} z_{k, m} \Lambda(m, n)\right. \\
& \left.\times \exp \left(-2 \pi j m \alpha \Delta f\left(k T+\tau_{l}\right)\right)\right] \\
& =\sum_{m=0}^{N-1} z_{k, m} h_{k, m} \Lambda(m, n),
\end{aligned}
$$

where $h_{k, m}=\sum_{L} \gamma_{l} \exp \left(-2 \pi j m \alpha \Delta f\left(k T+\tau_{l}\right)\right)$ is the multipath channel frequency response (CFR) acting on the $m^{t h}$ subcarrier of the $k^{t h}$ SEFDM symbol and $\Lambda(m, n)$ is the cross correlation between the subcarriers $m$ and $n$ given by [12]

$$
\Lambda(m, n)=\exp (j \pi \alpha(m-n)) \operatorname{sinc}(\alpha(m-n)) .
$$

A high level transceiver design is shown in Fig. 1 to give a general idea about CFR estimation for SEFDM signals over a multipath channel. In this work, a cyclic prefix of sufficient length is added to validate the assumption of null inter-symbol interference (ISI) between adjacent SEFDM symbols.

It is convenient to describe the transceiver process in the digital domain through a linear model. A demodulated SEFDM symbol $\hat{\mathbf{z}}_{k}$ from (3) can be expressed in a matrix form as

$$
\hat{\mathbf{z}}_{k}=\boldsymbol{\Lambda} \mathbf{H}_{k} \mathbf{z}_{k}+\mathbf{w}_{k},
$$

where $\hat{\mathbf{z}}_{k}, \mathbf{z}_{k}$ and $\mathbf{w}_{k}$ are $\mathbb{C}^{N \times 1}$ vectors of the $k^{t h}$ demodulated, transmitted baseband symbols and the white noise, respectively. $\mathbf{H}_{k} \in \mathbb{C}^{N \times N}$ is a diagonal matrix, where its diagonal element $H_{n, n}$ is the CFR coefficient on the $n^{\text {th }}$ subcarrier of the SEFDM signal and $\boldsymbol{\Lambda} \in \mathbb{C}^{N \times N}$ is the subcarriers correlation matrix, whose elements are given in (4).

The dual distortion of SEFDM signals introduced by the inter-carrier interference (ICI) and multipath channel effects compounds the difficulty and reduces the accuracy of channel estimation, relative to those of OFDM systems where $\boldsymbol{\Lambda}$ is an identity matrix. In the following, frequency domain channel estimation schemes for SEFDM are described.

\section{Frequency Domain Channel ESTIMATION SCHEMES}

Let $\mathbf{p} \in \mathbb{C}^{N \times 1}$ be the pilot symbol with the same characteristics of $\mathbf{z}$. Then, following (5), the demodulated pilot can will be represented by

$$
\hat{\mathbf{p}}=\mathbf{\Lambda} \mathbf{h} \odot \mathbf{p}+\mathbf{w},
$$

where $\odot$ denotes an element-wise multiplication and $\mathbf{h}$ is the diagonal of $\mathbf{H}$.

The three schemes considered in this paper are introduced below. Scheme I is based on the time domain channel estimation scheme of [10], but in the frequency domain. Scheme II is similar to the estimation scheme of the optical experimental work of [8], whilst scheme III presents the new channel estimation scheme proposed in this paper.

\section{A. Scheme I: Interpolated Partial Channel Esti- mation (PCE)}

PCE is based on the observation that there are subsets within the SEFDM subcarriers that can be mutually orthogonal. For $\alpha=b / c$ where $b, c \in \mathbb{Z}, b<c, N>c$, there are $\lceil N / c\rceil$ mutually orthogonal subcarriers $\left(\left|\Lambda_{m, n}\right|=0\right)$ for $\alpha(m-n) \in \mathbb{Z}$, where $\lceil$.$\rceil is the ceiling function$ [10]. Thus, the pilot is sent only over the mutually orthogonal subcarriers while the rest of the subcarriers are set to zero during pilot transmission. This type of arrangement overcomes the SEFDM ill-conditioning problem [10], as the condition number of its correlation matrix $\boldsymbol{\Lambda}$ increases by reducing $\alpha$ and/or increasing $N$. 


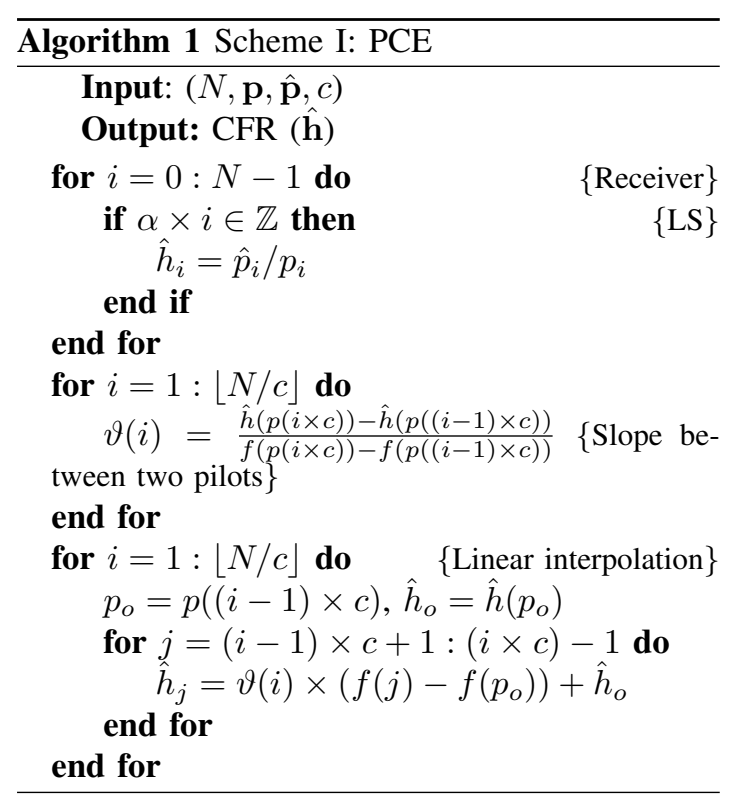

The first step to estimate the channel via PCE is LS estimation. Thereafter, the CFRs over the rest $(N-\lceil N / c\rceil)$ subcarriers are interpolated. For simplicity, linear interpolation is used. Algorithm 1 summarises the PCE algorithm for CFR estimation, where $f(p)$ is the frequency of the subcarrier modulated by the pilot $p$ and $\lfloor$.$\rfloor denotes the floor$ operation.

\section{B. Scheme II: OFDM Pilots with Interpolation}

Another way to solve the SEFDM illconditioning problem is using an OFDM pilot, as in [8]. SEFDM packs more subcarriers in the same bandwidth relative to OFDM. Thus, for the OFDM pilot, in order to occupy the same bandwidth as SEFDM, the number of subcarriers of the OFDM pilot $\left(N_{p}\right)$ for a specific $(\alpha)$ is given by

$$
N_{p}=\lceil\alpha \times N\rceil \text {, }
$$

where the transmitted OFDM pilot can be presented as

$$
p(t)=\sum_{n=0}^{N_{p}-1}\left(p_{n} \exp (2 \pi j n \Delta f t) .\right.
$$

For $\alpha=b / c$ where $b, c \in \mathbb{Z}, b<c, N>c$, there are $\lceil N / c\rceil$ subcarriers out of $N_{p}$ with an integer multiple of $(\alpha \Delta f)$. Hence, the CFRs on these subcarriers can be found directly by LS estimation. Thereafter, the CFRs on the rest $(N-\lceil N / c\rceil)$ are obtained through interpolation. Algorithm 2 summarises scheme II algorithm for CFR estimation.

\section{Scheme III: OFDM Pilots without Interpola- tion}

The previous method of OFDM pilot utilization shows the advantage of solving the SEFDM

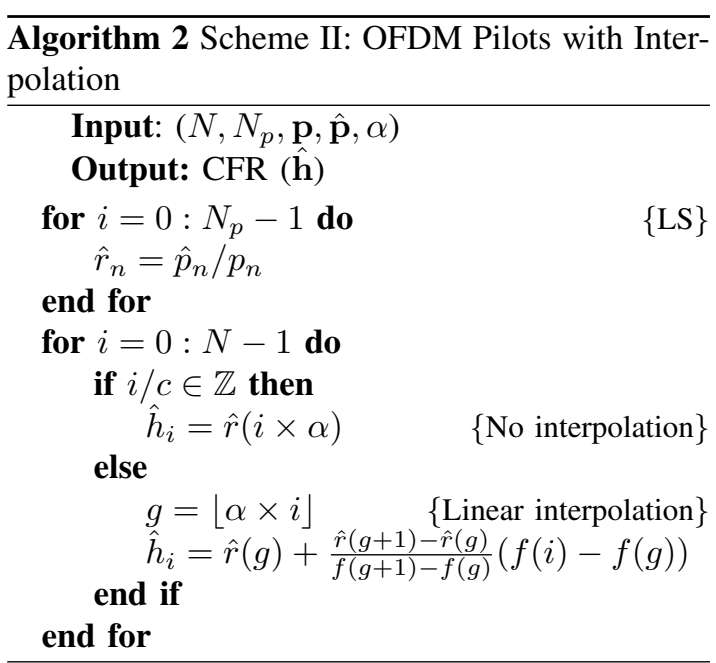

ill-conditioning problem. However, the required frequency-domain interpolation results in increasing the estimation scheme complexity and a dependency on $\alpha$.

To overcome the interpolation drawback, the main aim of the proposed scheme is to have an OFDM pilot symbol that occupies the same bandwidth of the SEFDM information symbols in (1), with the same number of subcarriers $N$ and frequency separation. These requirements, if satisfied, result in estimating the channel directly via $\mathrm{LS}$ without interpolation as given below

$$
\hat{\mathbf{h}}=\hat{\mathbf{p}} \cdot / \mathbf{p},
$$

where ./ denotes an element-wise division. Hence, to maintain the orthogonality of the pilot with a subcarreirs separation $\Delta f=\alpha / T$, the OFDM pilot symbol duration $\left(T_{p}\right)$ increases by a factor of $(1 / \alpha)$, such as $T_{p}=T / \alpha$.

To have a better understanding, Fig. 2 compares an OFDM pilot of duration $T_{p}$, with an SEFDM information symbol of duration $T$ for the case of $\alpha=0.8$. This figure presents the real part of the first three subcarriers $\left\{f_{n}=0, \Delta f, 2 \Delta f\right\}$ in (1). Clearly, unlike SEFDM, the OFDM pilot has to

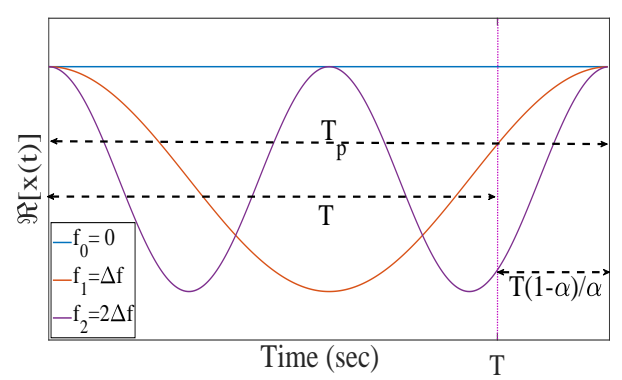

Fig. 2: The real part of $x_{k}(t)$ in the time domain for $f_{n}=0, \Delta f, 2 \Delta f$ with $\alpha=0.8$. 


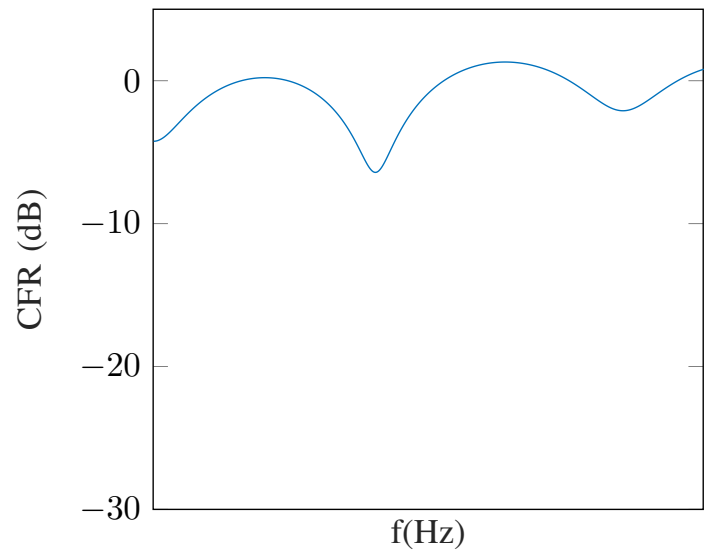

(a) Channel 1

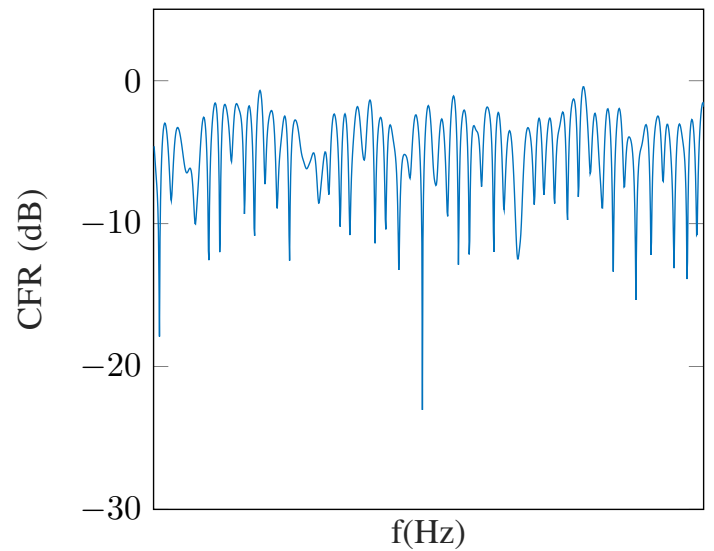

(b) Channel 2

Fig. 3: Channel frequency responses for channels $1 \& 2$.

maintain an integer number of cycles to preserve its orthogonality. Therefore, to complete a cycle, extra samples to cover the $T(1-\alpha) / \alpha$ overhead are needed, resulting in an extended pilot symbol period.

The added redundancy of this scheme compared to others depends on how frequent the pilot is sent. For instance, if the pilot is sent every $G$ information symbols, then, the added redundancy is $((1-\alpha) /(G+1)) \times 100 \%$. In the following discussion, and for the purpose of illustrating the utility of this new channel estimation scheme, two scenarios for slow varying or static channels, where a pilot symbol is sent ahead of information symbols, are presented. For fast varying channels, the same technique may be applied but with more frequent pilot transmission, in line with other mobile system practices.

\section{Performance \& Complexity Analysis}

Numerical simulations of the system of Fig. 1 are considered here for two channel scenarios. Channel 1 is the static channel in [13], which has been used before in [10] for SEFDM system with impulse response given by

$$
\begin{gathered}
h(t)=0.8765 \delta(t)-0.2279 \delta\left(t-T_{q}\right)+ \\
0.1315 \delta\left(t-5 T_{q}\right)-0.4032 e^{\frac{i \pi}{2}} \delta\left(t-7 T_{q}\right),
\end{gathered}
$$

where $\delta($.$) is the Dirac delta function and T_{q}=$ $T / Q$ is the sample time-duration in seconds and $Q=2 N$ is the number of time samples per SEFDM symbol.

Channel 2 is a quasi static Rayleigh channel that does not significantly change within an SEFDM symbol transmission but is time variant over the duration of the transmission of few symbols. Table. I summarises simulation parameters of channel 2, while Fig. 3 illustrates the CFRs for static channel 1 and a snapshot of channel 2 at a given time instant.
TABLE I: Channel 2 simulation parameters.

\begin{tabular}{|l|l|}
\hline Parameter & Value \\
\hline Number of delay taps & 8 \\
Maximum delay $(\tau)$ & $0.2 T<\tau<0.5 T$ \\
\hline
\end{tabular}

Based on (6), Fig. 1 and the algorithms discussed above, the received pilot symbol $\hat{\mathbf{p}}$ is used to calculate the estimated CFR $\hat{\mathbf{h}}$. Thence, $\hat{\mathbf{h}}$ is used to equalise the channel effects on the SEFDM information symbols via a joint equalisation and detection method similar to that implemented in [14]. Details of the joint equalisation and detection method are not included as the main focus of this paper is on CFR estimation. In this work, the mean square error (MSE) is used to evaluate the performance of the schemes considered. The MSE is given by

$$
\mathrm{MSE}=\mathbb{E}\left\{[\hat{\mathbf{h}}-\mathbf{h}]^{\mathrm{H}}[\hat{\mathbf{h}}-\mathbf{h}]\right\},
$$

where $\mathbb{E}[\cdot]$ is the expectation operator and $(\cdot)^{\mathrm{H}}$ is the Hermitian operator.

The simulation results of Fig. 4 show the MSE versus the energy per bit to noise ratio $\left(E_{b} / N_{0}\right)$ in $\mathrm{dB}$ for the three CFR estimation schemes and the two channels explored in this paper (top figures for channel 1 and bottom for channel 2). The comparison is held for three different (4-QAM mapped) pilot sizes $(N=16,128,1024)$ and two compression factors (i.e. $\alpha=0.8,0.67$ ).

The main conclusions that can be drawn from the results of Fig. 4 are: First, the performance of the first two schemes is enhanced by increasing $N$, and is a function of $\alpha$ and the flatness of the CFR. Second, due to the interpolation implemented in the first two schemes, they outperform the third scheme for the case of channel 1 with $N=128,1024$. This is because the AWGN noise is assumed to have zero mean, thus, interpolation helps in reducing the noise effect leading to a more accurate channel estimation. Finally, the advantage 


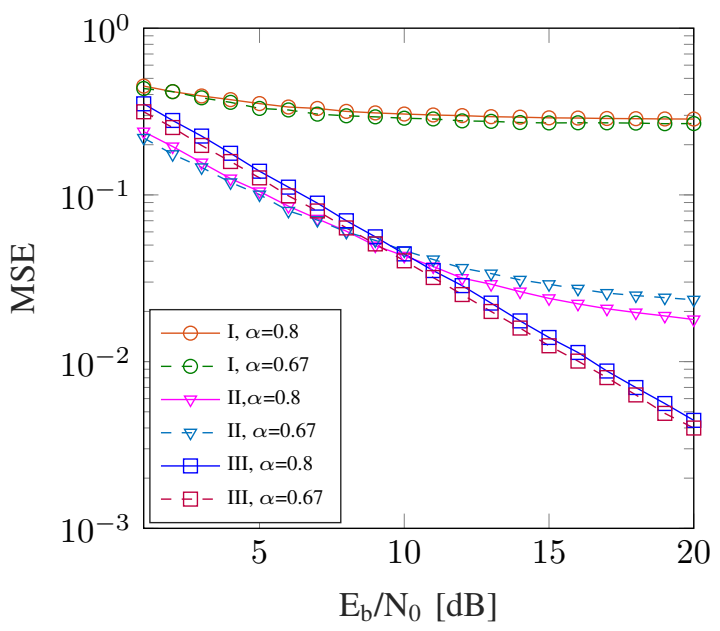

(a) Channel $1, N=16$

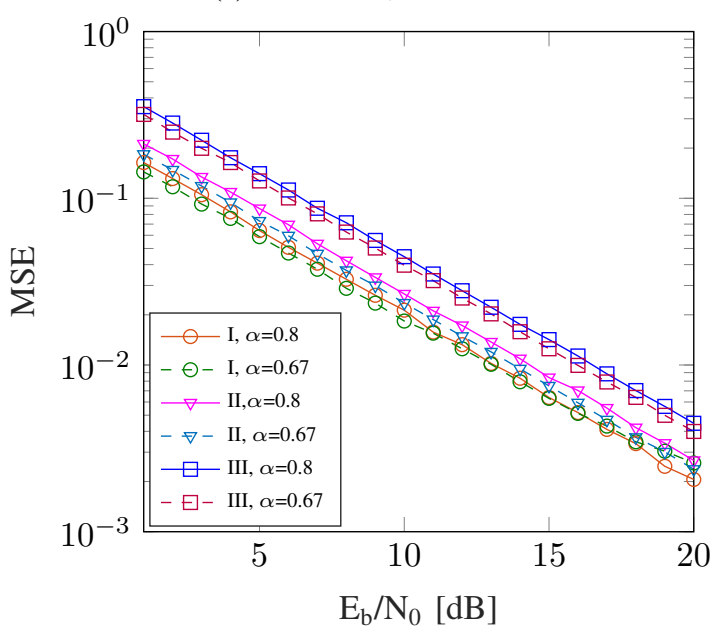

(c) Channel 1, N=1024

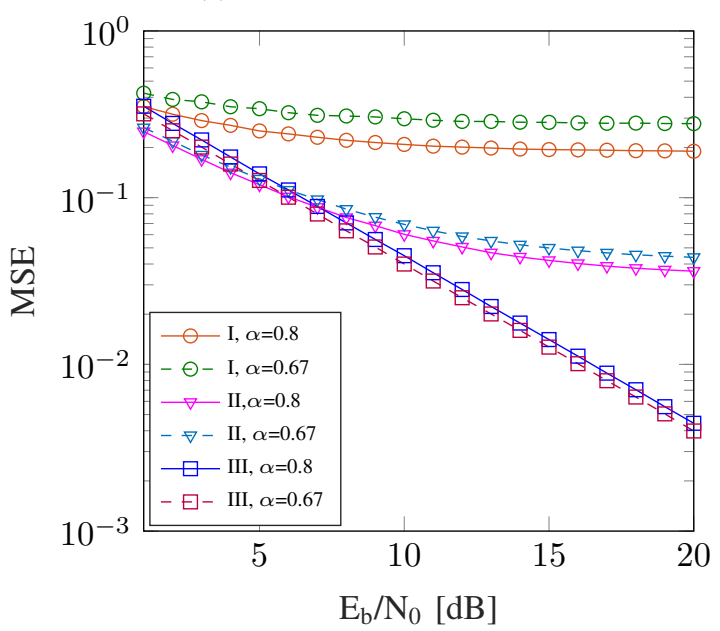

(e) Channel 2, $N=128$

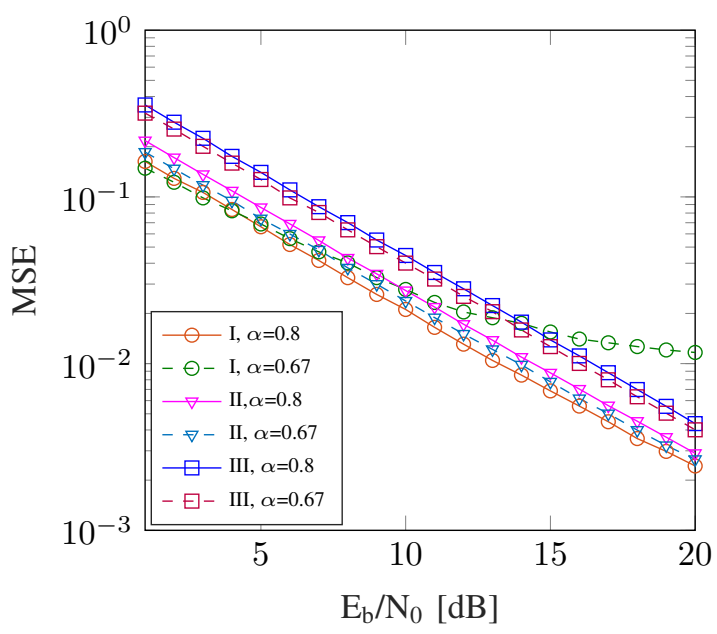

(b) Channel 1, $N=128$

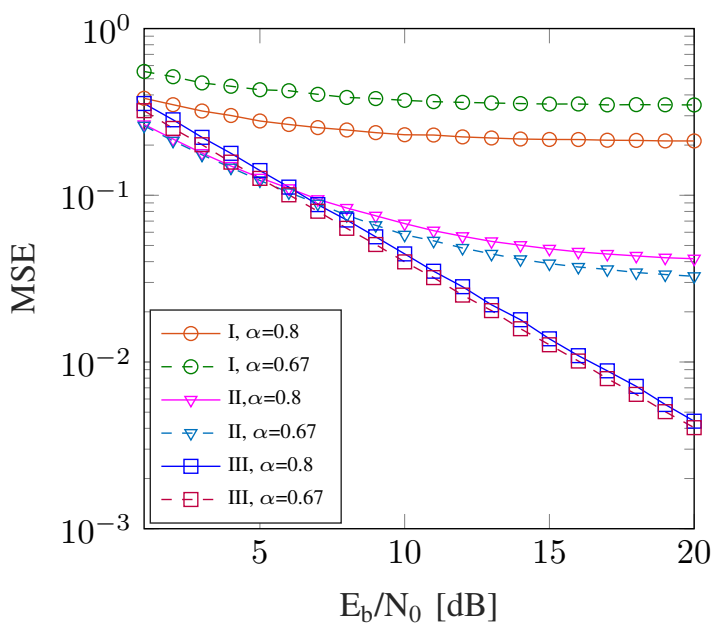

(d) Channel 2, $N=16$

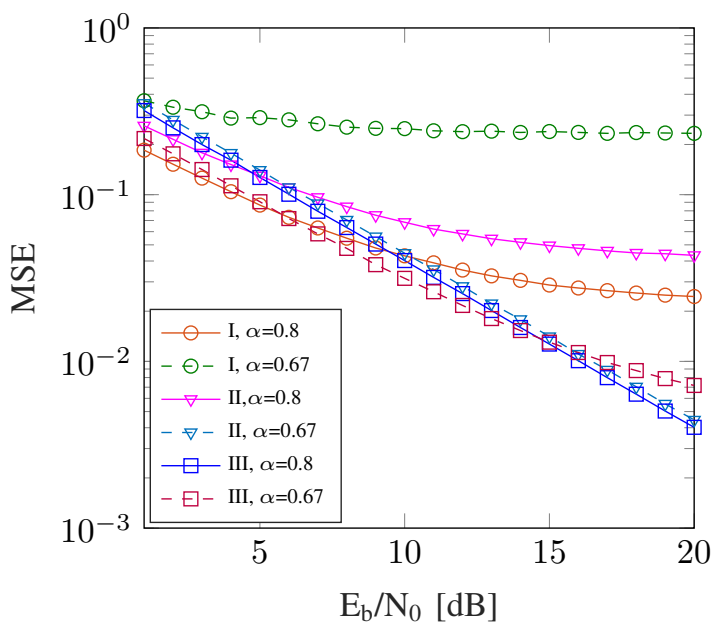

(f) Channel 2, $N=1024$

Fig. 4: MSE results for the three estimation schemes over channel $1 \&$ channel 2, for different values of $N$ and $\alpha$. 
TABLE II: Computational complexities (in terms of number of complex operations) for the three channel estimation schemes.

\begin{tabular}{|c|c|c|}
\hline & Multiplications & Additions \\
\hline Scheme I & $N+\lceil N / c\rceil-1$ & $2(N-1)$ \\
Scheme II & $2(N-\lceil N / c\rceil)+\lceil N \times c\rceil-1$ & $4(N-\lceil N / c\rceil)-1$ \\
Scheme III & $N$ & 0 \\
\hline
\end{tabular}

of scheme III can be seen from its enhanced performance that is independent of $N, \alpha$ and the channel condition.

Besides performance, complexity is another prime factor to consider. The total number of operations for the three schemes discussed above are counted using Algorithm 1,2 and equation (9). Table II summarises the complexity of all schemes in terms of the number of complex additions and multiplications. Clearly, scheme III has the lowest complexity.

We emphasize here that the complexities of scheme I and II depend on $\alpha$ unlike scheme III. Thus, the performance and complexity of scheme III make its implementation practical.

\section{CONCLUSIONS}

In this work, we devise a robust frequencydomain channel estimation scheme for SEFDM signals, where the transmitted block is divided into orthogonal and non-orthogonal multiplexing regions. Specifically, the pilot is sent over orthogonally spaced subcarriers as an OFDM symbol, while information symbols are sent over SEFDM; both using the same number of subcarriers and the same subcarrier spacing. Therefore, this new scheme does not require channel interpolation and results in accurate channel estimation, at the expense of slightly increased pilot duration overhead. The MSE results demonstrate that this new scheme maintains an accurate estimation of the channel regardless of the compression factor of SEFDM, the number of subcarriers and channel state. In addition, substantial reduction in computational complexity and implementation simplicity are achieved compared to other schemes.

\section{REFERENCES}

[1] F. Luo and C. Zhang, Signal Processing for 5G: Algorithms and Implementations, ser. Wiley - IEEE. Wiley, 2016.

[2] Z. K. Xiang, W. and X. S. Shen, Eds., 5G Mobile Communications, ser. Sprigner. Springer, 2017.

[3] M. Rodrigues and I. Darwazeh, "A Spectrally Efficient Frequency Division Multiplexing Based Communications System," in Proc. 8th Int. OFDM Workshop, Hamburg, 2003, pp. 48-49.

[4] T. Xu and I. Darwazeh, "Transmission Experiment of Bandwidth Compressed Carrier Aggregation in a Realis tic Fading Channel," IEEE Trans. Veh. Technol., 2016.

[5] T. Xu, S. Mikroulis, J. E. Mitchell, and I. Darwazeh, "Bandwidth Compressed Waveform for 60-GHz Millimeter-Wave Radio over Fiber Experiment," $J$. of Lightwave Technol., vol. 34, no. 14, pp. 3458-3465, July 2016
[6] Y. Wang, Y. Zhou, T. Gui, K. Zhong, X. Zhou, L. Wang, A. P. T. Lau, C. Lu, and N. Chi, "SEFDM Based Spectrum Compressed VLC System Using RLS Time-domain Channel estimation and ID-FSD Hybrid Decoder,' in ECOC 2016; 42nd European Conference on Optical Communication, Sept 2016, pp. 1-3.

[7] Y. Wang, T. G. Y. Zhou, K. Zhong, X. Zhou, L. Wang, C. L. A. P. T. Lau, and N. Chi, "Efficient MMSESQRD-Based MIMO Decoder for SEFDM-Based 2.4Gb/s-spectrum-Compressed WDM VLC System," IEEE Photonics Journal, vol. 8, no. 4, pp. 1-9, Aug 2016.

[8] D. Nopchinda, T. Xu, R. Maher, B. C. Thomsen, and I. Darwazeh, "Dual Polarization Coherent Optical Spectrally Efficient Frequency Division Multiplexing," IEEE Photon. Technol. Lett, vol. 28, no. 1, pp. 83-86, Jan 2016.

[9] M. Jia, Z. Yin, Q. Guo, G. Liu, and X. Gu, "Downlink Design for Spectrum Efficient IoT Network," IEEE Internet Things J., vol. PP, no. 99, pp. 1-1, 2017.

[10] S. Isam and I. Darwazeh, "Robust Channel Estimation for Spectrally Efficient FDM System," in 2012 19th International Conference on Telecommunications (ICT), April 2012, pp. 1-6.

[11] T. Ishihara and S. Sugiura, "Iterative frequency-domain joint channel estimation and data detection of fasterthan-nyquist signaling," IEEE Transactions on Wireless Communications, vol. 16, no. 9, pp. 6221-6231, Sept 2017.

[12] S. Isam and I. Darwazeh, "Characterizing the Intercarrier Interference of Non-orthogonal Spectrally Efficient FDM System," in 2012 8th International Symposium on Communication Systems, Networks Digital Signal Processing (CSNDSP), Poznan, July 2012, pp. 1-5.

[13] X. Wang, P. Ho, and Y. Wu, "Robust channel estimation and ISI cancellation for OFDM systems with suppressed features," IEEE Journal on Selected Areas in Communications, vol. 23, no. 5, pp. 963-972, May 2005.

[14] H. Ghannam and I. Darwazeh, "SEFDM over satellite systems with advanced interference cancellation," IET Communications, vol. 12, no. 1, pp. 59-66, 2018. 